\title{
NOTE ON THE SC LINEAR COLLIDER TESLA CAVITY DESIGN
}

\author{
J. Sekutowicz, D. Proch, C. Tang, DESY-MHF, 22603 Hamburg, FRG
}

\section{Abstract}

The experience we have gained over the last few years from experiments with superconducting cavities for the TESLA test facility, justifies a revision of the design proposed almost five years ago [1,2]. The proposed new design, presented here, takes the advantage of the high quality factor $\mathrm{Q}_{0}>10^{10}$ and low electron emission at the specified accelerating field of $25 \mathrm{MV} / \mathrm{m}$, as demonstrated by some tested cavities. The main aim of the design is to simplify production and preparation of superconducting (sc) cavities and thus to reduce the cost of the linear collider. The new cavity shape has an enlarged iris diameter with the following advantages: significantly lower loss factors, a simplified and less expensive scheme for the HOM damping and the suitability of hydroforming and higher stability of the field profile.

\section{INTRODUCTION}

The quality factor, $Q_{o}$, vs. $E_{\text {acc }}$ of two $1.3 \mathrm{GHz}, 9$-cell TESLA cavities is shown in Fig. 1. The $\mathrm{Q}_{0}$ reaches a very high value $\sim 5 \cdot 10^{10}$ at low field region and stays above $1.7 \cdot 10^{10}$ for one cavity and above $3 \cdot 10^{10}$ for the second one, for the accelerating field of $E_{\text {acc }}=25 \mathrm{MV} / \mathrm{m}$, the specified value for the sc linear collider [3]. The power dissipated in the cavity wall $\mathrm{P}_{\text {diss }}$, cooled by $2 \mathrm{~K}$

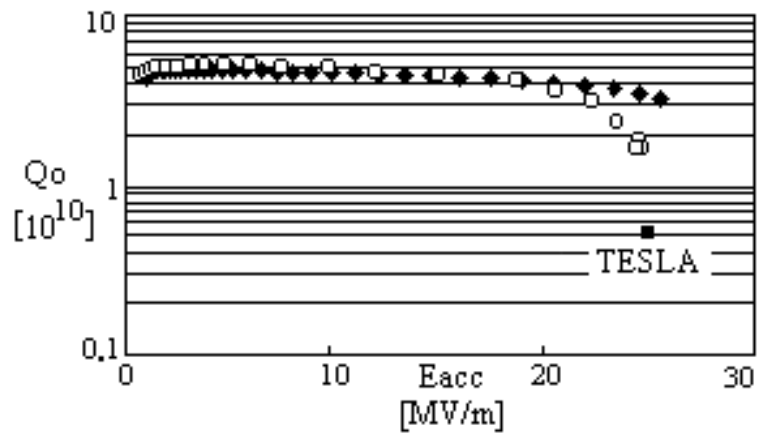

Fig. $1 \mathrm{Q}_{0}$ vs. $\mathrm{E}_{\text {acc }}$ as measured in a vertical cryostat

$\mathrm{LHe}$, is inverse proportional to $(\mathrm{R} / \mathrm{Q}) \cdot \mathrm{Q}_{0}$, where $(\mathrm{R} / \mathrm{Q})$ is the characteristic impedance of a cavity. For cavities with quality factors depicted in Fig. 1, the $P_{\text {diss }}$ at 25 $\mathrm{MV} / \mathrm{m}$ is 3.4 and 6 times respectively, lower than for a cavity with the specified value of $\mathrm{Q}_{0}=5 \cdot 10^{9}$ [3]. The small slope of both curves demonstrates the important feature that cavities have low electron emission. It results from careful cleaning during the cavity preparation and the low field enhancement factor $E_{\text {peak }} / E_{\text {acc. }}$. The shape of the TESLA structure, proposed almost five years ago, has been chosen to minimize: $\mathrm{E}_{\text {peak }} / \mathrm{E}_{\mathrm{acc}}$ to keep electron emission loading low and $\mathrm{H}_{\text {peak }} / \mathrm{E}_{\text {acc }}$ to shift quench level towards higher $\mathrm{E}_{\text {acc }}$ and to maximize (R/Q) to provide low cryogenic load. Final values of these parameters (see Table 1) have been achieved by means of a small iris diameter, but there are few negative consequences of the small aperture.

\section{WEAK POINTS OF THE CURRENT DESIGN}

The error of the field amplitude in an individual cell for the accelerating $\pi$ mode is proportional to $(\mathrm{N})^{2} / \mathrm{k}$, where $\mathrm{N}$ is the number of cells in the structure and $\mathrm{k}$ is the coupling. Since the cell-to-cell coupling $\mathrm{k}$ is small, the profile of the accelerating field is sensitive to the frequency perturbation of an individual cell. Any mechanical, chemical or thermal preparation of TESLA structures causes a perturbation of the field configuration. Many TTF cavities from the first production had to be re-tuned several times to keep the field unflatness below the specified level of $5 \%$. Since tuning had to be performed outside the clean room, each re-tuning required that the cavity must be re-cleaned. This makes the preparation more expensive and more time-consuming to an unacceptable level for 20000 sc cavities. After the helium vessel is welded, no access to the cells is possible and therefore re-tuning, even when necessary, is impossible.

Field unflatness decreases the effective accelerating field. Since the energy of colliding beams has been fixed, a compensation must be made for the lower effective gradient, either with additional length of the collider or by an operation of some cavities at $E_{\text {acc }}$ above $25 \mathrm{MV} / \mathrm{m}$. This requires only negligible additional RF power but the operation at a higher gradient may lead to an increased $\gamma$-radiation and a higher probability of quenches.

The second complication coming from the field unflatness is a difference in $\mathrm{Q}_{\text {ext. }}$ The acceleration process in the TESLA collider is performed during the transient. This requires all cavities to be at $25 \mathrm{MV} / \mathrm{m}$ at the same time, $533 \mu$ s after the RF pulse is switched on. As the cavity fill time depends on the loaded Q (in case of sc cavities mainly on $Q_{\text {ext }}$ ) differences in $Q_{\text {ext }}$ yield to differences in the accelerating gradient during acceleration. This means that the energy gain, in cavities with non nominal $\mathrm{Q}_{\text {ext }}$, changes from the beginning to the end of the pulse. To avoid this source of energy spread two adjustable fundamental mode (fm) coupler designs, based on a coaxial line technique, have been proposed [4,5]. In both designs, the adjustment of $Q_{\text {ext }}$ is performed by a change of penetration of the inner conductor in the beam tube. The adjustment unfortunately makes the design more complicated and expensive. Additionally, a fast voltage control loop will be used to reduce the energy spread, but this will require more RF power. 
A third problem resulting from the field unflatness is an increase of characteristic impedance of other modes, belonging to the fm passband. If the cavity is well tuned, then the characteristic impedances of these modes are negligible. Perturbation of an individual cell causes that especially the impedance of the mode $8 \pi / 9$ increases. The damping of these parasitic modes is possible only by $\mathrm{fm}$ coupler. So, it is expected that the external Q's of these modes are in the range of the nominal value of $\mathrm{Q}_{\text {ext }}$ of the fm. A typically observed field unflatness of $15 \%$ increases (R/Q) of the $8 \pi / 9$ mode from almost 0 to $5 \Omega$. In this case the impedance of this mode is 15 $\mathrm{M} \Omega$, as high as the impedance of monopole modes from the $\mathrm{TM}_{011}$ passband with the highest $(\mathrm{R} / \mathrm{Q}) \cong 150 \Omega$.

In the present design, neighboring cavities in a cryomodule are separated by a $3 / 2 \lambda$ long beam tube. Therefore the ratio of the active length to the total length of the TESLA structure is only 0.75 . Thus one quarter of both linacs length, $7 \mathrm{~km}$, is not used for the acceleration. The effective average accelerating field is then reduced from $25 \mathrm{MV} / \mathrm{m}$ to almost $19 \mathrm{MV} / \mathrm{m}$. One can improve the fill factor by increasing the number of cells per cavity and by shortening the interconnections between the cavities. Since the field profile of the present TESLA structures is already sensitive to frequency errors, an increase of $\mathrm{N}$ is impossible, so only interconnections could be made shorter to improve the fill factor.

The cost of the electron beam welding is a significant part of the cavity fabrication cost. Eighteen half cells and two beam tubes per cavity must be specially prepared to provide nineteen high quality welds. Alternative fabrication methods are under consideration to lower the production costs. Two of them hydroforming [6] and spinning [7], show a strong potential. The hydroforming of $\mathrm{Nb}$ cavities needs still more $\mathrm{R} \& \mathrm{D}$, but the experience with $\mathrm{Cu}$ cavities proofs that the required shape can be obtained with high precision and good reproducibility. The hydroforming process becomes simpler when the ratio of the maximum radius (equator) to the minimum radius ( iris) of a cavity is 2 or less. In the current design this ratio is almost equal to 3. The shape of the cavity plays also an important role. Also here, the tests performed on copper cavities showed that any narrow or small curvature, especially in the iris region, makes hydroforming more complicated. Unfortunately this is the case for the current cavity design.

The very high intensity FEL $(\lambda=1 \AA$ ) is one of the proposed new applications of the $3 \mathrm{~km}$ part of the TESLA e-linac. This operation requires very short bunches with $\sigma_{\mathrm{z}}=25 \mu \mathrm{m}$, which can excite wake fields to the $\mathrm{THz}$ region. Since the longitudinal loss factor $\mathrm{k}_{\|}$and thus the energy loss scale approximately with $\left(\sigma_{z}\right)^{-1 / 2}$, the total energy deposited by single bunch will be big. This will influence the beam. Additionally, the high energy photons may brake Cooper pairs in the superconducting material and increase the wall losses of the fm. As a remedy, to keep the energy deposition low, one can decrease $\mathrm{k}_{\|}$by enlarging the iris opening.

\section{NEW SHAPE}

We expect that the improved cavity shape should :

-increase cell-to-cell coupling,

-simplify hydroforming or spinning of the structure,

-reduce the loss factor $\mathrm{k}_{\|}$,

-simplify HOM mode damping,

- simplify the design of the fm coupler (not variable), -increase the ratio of the active length to the total length. Besides, the new design should keep, as much as possible, the advantages of the current shape. These advantages are parameters like: $(\mathrm{R} / \mathrm{Q}), \mathrm{E}_{\text {peak }} / \mathrm{E}_{\text {acc }}$ and $\mathrm{H}_{\text {peak }} / \mathrm{E}_{\text {acc }}$. Some of the disadvantages we have pointed out can be overcome with an increase of the iris diameter [2]. The proposed new shape (Fig. 2) has an iris aperture of 102 $\mathrm{mm}$ The iris is circularly formed with $\mathrm{r}=17 \mathrm{~mm}$. The parameters of the old and the new shape are listed in Table 1.

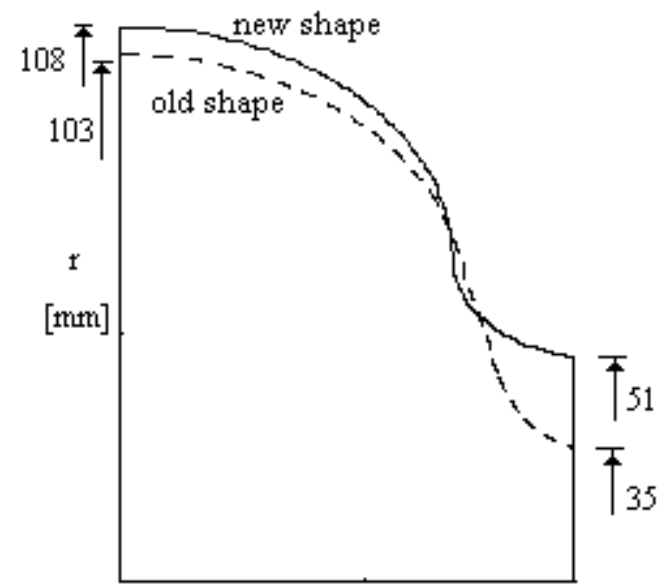

Figure 2 The new and the old shape of the inner cell (not in scale)

Table 1 Parameters of the old and the new cavity shape

\begin{tabular}{|c|c|c|c|}
\hline Parameter & Old & New & Change [\%] \\
\hline$(\mathrm{R} / \mathrm{Q})$ & 1035 & 695 & -33 \\
\hline$[\%]$ & 1.84 & 5.51 & 200 \\
\hline $\mathrm{E}_{\text {peak }} / \mathrm{E}_{\mathrm{acc}}$ & 2.0 & 2.34 & 17.0 \\
\hline $\mathrm{H}_{\text {peak }} / \mathrm{E}_{\mathrm{acc}} \quad[\mathrm{Oe} /(\mathrm{MV} / \mathrm{m})]$ & 42.6 & 50.2 & 17.8 \\
\hline $\mathrm{k}_{\perp}(\sigma=1 \mathrm{~mm})[\mathrm{V} /(\mathrm{pCm})]$ & 18 & 6.7 & -63 \\
\hline $\mathrm{k}_{\|}(\sigma=1 \mathrm{~mm}) \quad[\mathrm{V} / \mathrm{pC}]$ & 8.7 & 5.5 & -37 \\
\hline iris diameter & 70 & 102 & 46 \\
\hline $\begin{array}{l}\text { iris shape } \\
\text { h./v. axis, radius [mm] }\end{array}$ & $\begin{array}{l}\text { ellip. } \\
12 / 19\end{array}$ & $\begin{array}{c}\text { circle } \\
17\end{array}$ & \\
\hline $\mathrm{r}_{\text {equ }} / \mathrm{r}_{\text {iris }} \quad$ inner /end cell & $3 / 2.7$ & $2 / 1.9$ & $-29 /-27$ \\
\hline
\end{tabular}

\section{DISCUSSION}


The cell-to-cell coupling increased by a factor of three as compared to the current design. This makes the structure less sensitive to any machining tolerances and provides room to increase the number of cells per cavity. The new geometry is more suitable for hydroforming or spinning. The ratio of the equator radius to the iris radius is near 2 for the inner cell and even below 2 for the end cell. The iris region is now wider and the wall can be made thicker in this region. Hereby stiffening rings, used in the current design to reduce the Lorentz force detuning, can be avoided [6].

The loss factors of the new geometry are significantly lower. The single passage deposited energy is reduced by $37 \%$ for the monopole modes and by $63 \%$ for the dipole modes. The decrease of the loss factors indicates a decrease of HOMs characteristic impedances, too. Therefore the energy deposited in the resonantly excited parasitic modes will be reduced. This gives more relaxed requirements for the damping of these modes.

The enlarged iris of the new end cell enables an increase in diameter of the beam tube. We have fixed this diameter to $0.11 \mathrm{~m}$. It is the smallest size that allows propagation of all monopole modes in the tube. The first eight dipole modes stay under cutoff but their field in the beam tube seems to be high enough for damping to the BBU limit. Two HOM couplers shifted angularly by $90^{\circ}$ and attached at every second beam tube may be used for the damping of parasitic modes excited in two neighboring cavities. This damping scheme requires only one HOM coupler per cavity. Due to the HOM propagation the distance between HOM coupler and end cells can be much larger than for the current design. Since the fundamental mode is under cutoff and the distance to end cells is large, a very little fm rejection, achievable without any additional filter, is needed to prevent output cables and feedthroughs from overheating. This simplifies the design of the HOM coupler.

As the field pattern is stable, variable couplers are not necessary. If operation of the collider should differ from the nominal one, $Q_{\text {ext }}$ can be changed by means of a 3stub waveguide transformer installed in the input line of each cavity. The transformer also makes the RF distribution system more flexible for phase adjustment and gives more freedom in the length of intersections.

There are mainly two negative consequences of the larger iris aperture. As predicted, a larger aperture lowers $(\mathrm{R} / \mathrm{Q})$ of the $\mathrm{fm}$. The reduction is compensated by a high $\mathrm{Q}_{\mathrm{o}}$ and finally the cryogenic loss is smaller. In order to keep cavities matched for the nominal operation: $U=26$ MV/cavity and the $\mathrm{I}_{\mathrm{b}}=8 \mathrm{~mA}, \mathrm{Q}_{\mathrm{ext}}$ should be increased by $33 \%$, and fill time will therefore be longer by $240 \mu$ s. When $\mathrm{Q}_{\text {ext }}$ has still its previous nominal value, $2 \%$ of the RF power will be reflected but fill time will be longer by $180 \mu$ s. This problem should be investigated in more detail. The ratios of $\mathrm{E}_{\text {peak }} / \mathrm{E}_{\text {acc }}$ and $\mathrm{H}_{\text {peak }} / \mathrm{E}_{\text {acc }}$ have increased by $17 \%$. This means more $\gamma$-radiation at the specified $\mathrm{E}_{\mathrm{acc}}$ and higher quench probability. Surface cleaning methods (BCP, high pressure water rinsing) and assembling methods are still in an optimization phase. Also inspection methods of the $\mathrm{Nb}$ material, which help to eliminate $\mathrm{Nb}$ sheets with defects leading to quenches, are under development. It is the hope that further progress in the fabrication, the assembly and the cleaning methods can compensate for the increase of these parameters.

\section{CONCLUSION}

Table 2 Summary of features of the new shape

\begin{tabular}{|l|c|}
\hline $\mathrm{k}$, cell-to-cell coupling & + \\
\hline $\mathrm{k}_{\|}$, longitudinal loss factor & + \\
\hline $\mathrm{k}_{\perp}$, transversal loss factor & + \\
\hline $\mathrm{r}_{\text {equ }} / \mathrm{r}_{\text {ris }} \quad$ inner cell and end cell & + \\
\hline fill factor of both linacs & + \\
\hline simplified scheme for HOM damping & + \\
\hline simplified fm coupler & + \\
\hline$(\mathrm{R} / \mathrm{Q}) \quad f m$ characteristic impedance & - \\
\hline $\mathrm{E}_{\text {peak }} / \mathrm{E}_{\text {acc }}, \mathrm{H}_{\text {peak }} / \mathrm{E}_{\text {acc }}$ & - \\
\hline
\end{tabular}

A summary of the positive and the negative features of the new cavity shape is given in Table 2 . We plan to build copper models of the new structure to prove experimentally the stability of the accelerating field, the proposed scheme of the HOM damping and the coupling between structures for the fm and the HOMs. As a next step, $\mathrm{Nb}$ prototypes should be fabricated to investigate the hydroforming method, the Lorentz detuning and to check new limitations in the accelerating field.

\section{ACKNOWLEDGMENTS}

We would like to express our gratitude to the TESLA collaboration group for many helpful discussions.

\section{REFERENCES}

[1] E. Haebel, A. Mosnier, J.Sekutowicz, „Cavity Shape Optimization for Superconducting Linear Collider", Proc.of HEACC'92, Hamburg, 1992.

[2] E. Haebel, A. Mosnier, „Large or Small Iris Aperture in SC Multicell Cavities", Proc. of The $5^{\text {th }}$ SRF Work.,Hamburg, 1991.

[3] D. A. Edwards, ,TESLA TEST FACILITY LINACDesign Report“", TESLA Rep. 95-01.

[4] B. Dwersteg, ,,Mechanical Concept of TESLA Main Coupler Development at DESY“,TESLA Rep 93-15

[5] M.Champion, ,RF Input Coupler and Windows: Performances, Limitations and Recent Developments“, Proc. of $7^{\text {th }}$ Work. on S RF, Gif sur Yvette, 1995.

[6] H. Kaiser, DESY; private communication.

[7] V. Palmieri et al., ,,Recent Experience with the Spinning of $1.5 \mathrm{GHz}$ Seamless Copper Monocells“, Proc. of $7^{\text {th }}$ Work. on SRF, Gif sur Yvette, 1995. 